http://dx.doi.org/10.11646/phytotaxa.177.2.5

\title{
Pseudosymblepharis perlongifolia (Bryophyta, Pottiaceae), a neglected species from Malaysia new for America
}

\author{
MARTA ALONSO, MARÍA J. CANO \& JUAN A. JIMÉNEZ \\ Departamento de Biología Vegetal, Facultad de Biología, Universidad de Murcia, Campus de Espinardo, 30100- \\ Murcia, Spain. \\ E-mail: m.alonsogarcia@um.es
}

\begin{abstract}
The Asian moss Pseudosymblepharis perlongifolia, previously known only from Malaysia, is reported for the first time for America on the basis of several collections from Maricao (Puerto Rico). The principal distinctive characters that separate it from the nearest species of Pseudosymblepharis and related genera are discussed. The species is described and illustrated.
\end{abstract}

\section{Resumen}

El musgo asiático Pseudosymblepharis perlongifolia es citado por primera vez en América, en base a varias colecciones de Maricao (Puerto Rico). Hasta ahora, la especie era conocida exclusivamente de Malasia. En este trabajo se discuten los principales caracteres que permiten diferenciarla de otras especies de Pseudosymblepharis, así como de especies cercanas de otros géneros. La especie es descrita e ilustrada.

Key words: distribution, mosses, Puerto Rico, taxonomy

\section{Introduction}

In the context of a worldwide taxonomic revision of Pseudosymblepharis Brotherus (1924: 261), we studied the type material of Trichostomum perlongifolium Fröhlich (1962: 92). This species was described based on a single collection made by W. Meijer on Mount Kinabalu, Borneo, in the East Malaysian state of Sabah. Trichostomum perlongifolium was transferred to Pseudosymblepharis by Zander (1993). However, up to then, the species had been forgotten and was not even included in the Malesian moss flora (Eddy 1990). Sollman (2000), in his taxonomic revision of genus Pseudosymblepharis in Asia, reduced Pseudosymblepharis perlongifolia (J. Froeh1.) Zander (1993: 80) to synonymy with $P$. bombayensis (Müller 1851: 628) Sollman \& Een (1996: 2). In addition, he included under $P$. bombayensis the five species of Pseudosymblepharis known from Asia: P. angustata (Mitten 1859: 28) Hilpert (1933: 670), P. duriuscula (Mitten 1859: 27) Chen (1941: 153), P. indica Dixon \& P. de la Varde (1927: 166), P. khasiana (Mitten 1859: 29) Zander (1993: 80) and P. perlongifolia. Moreover, Sollman (2001) transferred P. bombayensis to Chionoloma bombayense (Müll.Hal.) Sollman (2001: 139), based on work carried out by Menzel (1992), so that, up to now, $P$. perlongifolia has been considered a synonym.

During the course of a revision of American Pseudosymblepharis (Alonso et al. 2014), we had the opportunity to examine several collections of Puerto Rican mosses deposited at NY. Among these collections, we found some specimens belonging to Pseudosymblepharis which did not match any previously known species of this genus in America. A detailed study of these specimens allowed us to identifiy them as P. perlongifolia.

This paper presents a complete description and LM photographs of this neglected species. Moreover, its distribution, habitat, and comparisons with closely related species are provided. 


\section{References}

Aiba, S. \& Kitayama, K. (1999) Structure, composition and species diversity in an altitude-substrate matrix of rain forest tree communities on Mount Kinabalu, Borneo. Plant Ecology 140: 139-157.

Allen, B. (1994) Moss flora of Central America, Part 1. Sphagnaceae-Calymperaceae. Monographs in Systematic Botany from the Missouri Botanical Garden 42: 1-242.

Allen, B. (2002) Moss flora of Central America, Part 2. Encalyptaceae-Orthotrichaceae. Monographs in Systematic Botany from the Missouri Botanical Garden 90: 1-699.

Alonso, M., Cano, M.J. \& Jiménez, J.A. (2014). A new combination in Pseudosymblepharis (Pottiaceae) from Central and South America. Journal of Bryology 36: 27-33.

http://dx.doi.org/10.1179/1743282013Y.0000000084

Andrews, A.L. (1920) Tortula caroliniana, new species. The Bryologist 23: 72-76.

Bardunov, L.V. (1969) Handbook of mosses of the Central Siberia. Hayka, Nauka, Leningrad, 329 pp.

Bartram, E.B. (1946) New species and new combinations of Guatemalan mosses. The Bryologist 49: 109-125.

http://dx.doi.org/10.2307/3239484

Brongniart, M. \& Gris, A. (1863) Notes sur deux genres nouveaux de Myrtacées de la Nouvelle-Calédonie. Bulletin de la Société Botanique de France 10: 369-374.

http://dx.doi.org/10.1080/00378941.1863.10827261

Brotherus, V.F. (1924) Musci (Laubmoose). 1 Hälfte. 10. In: Engler, H.G.A. \& Prantl, K.A.E. (Eds.) Die Natürlichen Pflanzenfamilien, ed. 2. Duncker \& Humblot, Berlin, 478 pp.

Chen, P.C. (1941) Studien über die ostasiatischen Arten der Pottiaceae, II. Hedwigia 80: 141-322.

Chiang, T.Y. \& Lin, S. (2001) Taxonomic revision and cladistic analysis of Diphyscium (family Diphysciaceae) of Taiwan. Botanical Bulletin of Academia Sinica 42: 215-222.

Crum, H.A. (1952) Pseudosymblepharis in Middle America. The Bryologist 55: 137-142.

Crum, H.A. \& Steere, W.C. (1956) Puerto Rican Musci. New species and new combinations. The Bryologist 59: $246-255$.

Deguchi, H. (1984) Diphyscium unipapillosum, sp. nov. (Diphysciaceae, Musci) from Japan. Journal of Japanese Botany 59: 97-103.

Dixon, H. \& Potier de la Varde, R. (1927) Contribution à la flore bryologique de 1'Inde méridionales. Archives de Botanique, Bulletin Mensuel 1: 161-184.

Eddy, A. (1990) A Handbook of Malesian Mosses. Vol. 2. Leucobryaceae to Buxbaumiaceae. Natural History Museum Publications, London, $256 \mathrm{pp}$.

Endlicher, S. (1847) Synopsis Coniferarum. Sangalli, apud Scheitlin \& Zollikofer, Cracoviae, 368 pp. http://dx.doi.org/10.5962/bhl.title.15336

Forster, J.R. \& Forster, J.G. (1775) Characteres generum plantarum: quas in itinere ad insulas Maris Austrails. B. White, T. Candell, \& P. Elmsly, London, $150 \mathrm{pp}$. http://dx.doi.org/10.5962/bhl.title.4448

Fröhlich, J. (1962) Musci novi malesiani collecti a Dre. Guil. Meijer. Revue Bryologique et Lichénologique 31: 91-94.

Gaertner, C. (1805) Supplementum carpologiae seu continuati operis Josephi Gaertner de fructibus et seminibus plantarum. Sumtibus Carol. Frid. Enoch Richter bibliopolae Lipsiensis, Leipzig, 128 pp.

http://dx.doi.org/10.5962/bhl.title.59684

Gaertner, J. (1788) De fructibus et seminibus plantarum Vol. 1. Typis Academiae Carolinae, Stutgardiae, 384 pp.

Grout, A.J. (1938) The moss Flora of North America North of Mexico. Grout, A.J., Newfane, Vermont, 264 pp.

Hilpert, F. (1933) Studien zur systematik der Trichostomaceen. Beihefte zum Botanischen Centralblatt 50: 585-706.

Jacquin, N.J. (1760) Enumeratio systematica plantarum, quas in insulis Caribaeis. Inter Documentation Company AG, Zug, Switzerland, $41 \mathrm{pp}$.

http://dx.doi.org/10.5962/bhl.title.737

Kiaerskov, H.F.C. (1890) Myrtaceae ex India occidentali a dominis Eggers, Krug, Sintenis, Stahl. Botanisk Tidsskrift 17: $248-492$.

Kitayama, K. (1992) An altitudinal transect study of the vegetation on Mount Kinabalu, Borneo. Vegetatio 102: 149-171. http://dx.doi.org/10.1007/bf00044731

Knoblauch, E.F. (1895) Wissenschaftliche Original-Mittheilungen. Zur Kenntniss einiger Oleaceen-Genera. Botanisches Centralblatt 61: 81-87.

Krug, C.W.L. \& Urban, I. (1895) Additamental ad cognitionem Indiae occidentalis. II. Botanische Jahrbücher für Systematik, Pflanzengeschichte und Pflanzengeographie 19: 577-681.

Lamarck, J.B. (1791) Tableau encyclopédique et méthodique des trois règnes de la nature, Botanique 1. Panckoucke, Paris \& Liège, 496 
pp.

http://dx.doi.org/10.5962/bhl.title.218

Lambert, A.B. (1807) A description of the genus Pinus 1. J. White, Horace's Head, Fleet John, London, 98 pp.

Laubenfels de, D.J. (1969) A revision of the Malesian and Pacific rainforest conifers, I. Podocarpaceae. Journal of the Arnold Arboretum 50: 315-369.

Mitten, W. (1859) Musci Indiae orientalis, an enumeration of the mosses of the East Indies. Journal of the Proceedings of the Linnean Society, Botany, Supplement 1: 1-96. http://dx.doi.org/10.1111/j.1095-8339.1859.tb02466.x

Mitten, W. (1869) Musci Austro-Americani. Journal of the Linnean Society, Botany 12: 1-659. http://dx.doi.org/10.1111/j.1095-8339.1871.tb00633.x

Mitten, W. (1891) An enumeration of all the species of music and hepaticae recorded from Japan. Transactions of the Linnean Society of London, Botany 3: 153-206. http://dx.doi.org/10.1111/j.1095-8339.1891.tb00626.x

Menzel, M. (1992) Nomenklatorische notizen zu Eddys "Handbook of Malesian Mosses” (Band 2). Willdenowia 22: 197-199.

Müller, C. (1851) Synopsis muscorum frondosorum omnium hucusque cognitorum, 2. A. Foerstner, Berlin, 772 pp. http://dx.doi.org/10.5962/bhl.title.31

Müller, C. (1857) Manipulus muscorum e Flora Novae Granadae. Botanische Zeitung (Berlin) 15: 577-583.

Müller, C. (1874) Novitates bryothecae Müllerianae. 3. Musci Mexicani praesertim a Cl. C. Mohr et Sartorius collecti. Linnaea 38: 620660.

Müller, F.A. (1829) Erstes Verzeichnis sardinischer Laubmoose, wie auch derjenigen welche von meinem Freunde Herrn Fleischer bei Smyrna aufgefunden worden sind, nebst Beschreibungen und Abbildungen einiger neuer Arten. Flora 12: 385-396.

Norris, D.H. (1981) Diphyscium chiapense, a new species from southern Mexico. The Bryologist 84: 375-378. http://dx.doi.org/10.2307/3242856

O'Shea, B.J. (2006) Checklist of the mosses of sub-Saharan Africa. Tropical Bryology Research Reports 6: 1-252.

Paris, É.G. (1894-1898) Index bryologicus sive enumeratio muscorum hucusque cognitorum adjunctis synonyma distributioneque geographica locupletissimis quem conscripsit E. G. Paris. Paul Klincksieck, Paris. http://dx.doi.org/10.5962/bhl.title.643

Pierre, J.B.L. (1891) Notes botaniques: Sapotacées, 2. Paul Klincksieck, Paris. http://dx.doi.org/10.5962/bhl.title.10315

Silander, S., Gil de Rubio, H., Miranda, M. \& Vazquez, M. (1986) Compendio enciclopédico de los recursos naturales de Puerto Rico. Tomo II. Departamento de Recursos Naturales, San Juan, Puerto Rico.

Sollman, P. (2000) A taxonomic revision of Pseudosymblepharis Broth. (Musci: Pottiaceae) in Asia. Tropical Bryology 18: $129-145$.

Sollman, P. (2001) A new combination in Chionoloma Dix. (Musci, Pottiaceae). Tropical Bryology 20: 139.

Sollman, P. \& Een, G. (1996) Mosses from the Mascarenes - 5. Tropical Bryology 12: 1-4.

Sprague, T.A. (1932) Miscelaneous notes. Bulletin of Miscellaneous Information Kew 1932: 350-352.

Tan, B.C. (1998) Noteworthy disjunctive patterns of Malesian mosses. In: Hall, R. \& Holloway, J.D. (Eds.) Biogeography and Geological Evolution of Southeast Asia. Backhuys, Leiden, pp. 235-241.

Urban, I. (1899) Symbolae Antillanae seu fundamenta Florae Indiae Occidentalis. Fratres Borntraeger, Lipsiae, 536 pp. http://dx.doi.org/10.5962/bhl.title.144

Vahl, M. (1796) Eclogae Americanae seu descriptiones plantarum praesertim Americae meridionalis, nondum cognitarum 1. Impensis auctoris, Copenhagen, $58 \mathrm{pp}$. http://dx.doi.org/10.5962/bhl.title.69096

Zander, R.H. (1993) Genera of the Pottiaceae: mosses of harsh environments. Bulletin of the Buffalo Society of Natural Sciences 32 : $1-378$. 